\title{
FDDI-Based Digital Audio Interfaces
}

\author{
U. Zölzer, N. Kalff \\ Telecommunications Group \\ Technical University of Hamburg-Harburg \\ Eissendorfer Str. 40, D-2100 Hamburg 90, FRG \\ Tel. 49407718 2166, EAX 494077182281
}

\begin{abstract}
Digital audio processing is an emerging field of different standards for consumer and professional applications. Besides three main sampling frequencies (professional $48 \mathrm{kHz}$, consumer $44.1 \mathrm{kHz}$, broadcasting $32 \mathrm{kHz}$ ) two transmission formats have been established, namely a two-channel serial transmission format (AES/EBUFormat) and a multichannel audio digital interface (MADI). The interface techniques between these adopted standards will be discussed considering synchronization problems and the hardware complexity.
\end{abstract}

\section{Introduction}

Interfacing different single digital audio systems can be accomplished via the AES/EBU serial transmission link which is a unidirectional two-channel connection running at a data rate up to $3.072 \mathrm{Mbits} / \mathrm{sec}$ [1]. A consumer interface format (IEC) is derived from the AES/EBU two-channel format. The MADI interface format allows the transmission of 56 audio channels with a data rate of $100 \mathrm{Mbits} / \mathrm{sec}$ [2]. This high-speed link is a unidirectional transmission based on a network specification called the Fiber Data Distributed Interface (FDDI).

The distribution of different AES/EBU two-channel signals and multichannel signals is specially needed in broadcasting and studio applications. A typical configuration of a studio complex is shown in Fig. 1.

The main systems are mixed analog and digital I/O-Systerns (ADIO) which contain $\mathrm{AD} / \mathrm{DA}$-converters, $\mathrm{AES} / \mathrm{EBU}$ input and output signals and two-channel systems connected via sampling frequency converters (SFC), digital distribution systems (DD), audio signal processing systems (ASP) and human interfaces. The signal processing systems ADIO, DD and ASP are connected by two-way multichannel audio digital interfaces. Each of these systems is synchronized to an inhouse master sampling clock avoiding sampling frequency conversions. For multichannel sources which can not be synchronized to a common reference clock sampling frequency conversion systems are needed.

Among the switching techniques synchronous data routing can be utilized by timedivision multiplexing and asynchronous data routing by traditional crosspoint systems. 
In the first part of this paper we will discuss the MADI format, FDDI solutions and the hardware complexity. In the second part we will focus on synchronization problems, sampling frequency conversions and their hardware complexity.

\section{FDDI and MADI}

\section{- FDDI (Fiber Data Distributed Interface)}

FDDI is a high-speed serial interface using fiberoptic cables. Being about 10 times faster than traditional links it serves as point to point connection between two computers or as a fast backbone for a local area network in a ring topology. A single FDDI-based link can carry up to $100 \mathrm{MBits} / \mathrm{sec}$ of user data. The actual transmission rate is $125 \mathrm{MBits} / \mathrm{sec}$. The redundancy is added by the transmitter to introduce two additional features. The first is error detection, allowing to recognize bit errors and mark illegal bytes. Second, the user may use up to 15 commands to control the receiver hardware. The conversion from the users data bytes being transmitted with up to $12.5 \mathrm{MBytes} / \mathrm{sec}$ into the coded bitstream with $125 \mathrm{MBits} / \mathrm{sec}$ and back is performed by AMD's [3] two TAXIchips (Transparent Asynchronous Transmitter/Receiver Interface), the Am7968 transmitter and the Am7969 receiver (see Fig. 2).

Data transmission is initiated by using the transmitters strobe pin. The receiver announces a received data byte with the data strobe pin. During intervals without actual data the transmitter sends a special pattern, the Sync command, to keep the the receivers phase-locked loop locked onto the incoming bit boundary. The chipset within two 28-pin PLCC devices can drive conventional coaxial cables as well as fiberoptic cables.

- MADI (Multichannel Audio digital interface)

MADI is a serial interface format which carries up to 56 channels of digital audio data with a sampling rate between $32 \mathrm{kHz}$ and $48 \mathrm{kHz} \pm 12.5 \%$. Using fiberoptic cables a transmission distance up to $2 \mathrm{~km}$ is possible, with coaxial cables about $50 \mathrm{~m}$. The channel format is based on the two-channel AES/EBU format, where each channel is represented by 32 bits labelled 0 to 31 (Fig. 3). 24 bits are used for the representation of the audio data. The MADI frame period consists out of the 56 channels which are transmitted sequentially starting with channel 0 , and ending with channel 56 . The description of bits 4-31 follows those used by the AES/EBU format. The first channel within a sampling period is marked by the first preambel bit. The second preamble bit defines the status of the associated channel to be active or not, the third preamble bit is used for marking a stereo signal and the fourth preamble bit marks a 192-frame block, which is used at the receiver to decode the status (C bit) and user data (U bit).

MADI is based on FDDI and so utilizes the AMD TAXIchips. MADI transports only the pure data and does not carry any information about the sampling frequency.

- Hardware Complexity

Routing the 56 channels from a time-division multiplexing parallel bus system to the transmitter can be accomplished by writing data continously into a dual-ported ram 
(Fig. 4). The composite side is connected to a routing controller which reads the 56 choosen channels. Then the channel data is handed to a multiplexer converting the 32 bit AES/EBU data into four bytes which are transferred to the Am7968. At the receiver a demultiplexer collects the four bytes from the Am7969 and converts it back into the AES/EBU format. Since there are only about 50 ns to pick up the data, an additional buffer is necessary allowing the routing controller to write the data into selected memory locations of a dual-ported ram. The other side of the dual-ported ram is controlled by a time-division multiplexing parallel bus system.

\section{Synchronization}

The synchronization of systems with different sampling rates is needed when two or more digital systems are brought together in digital distribution systems or digital processing systems (refer to Fig. 1). The first synchronization problem appears when systems are operating at nominally the same sampling rate, but only one system is synchronized to a master reference clock. The second synchronization situation is characterized by systems which are operating with different sampling rates $(32 \mathrm{kHz}, 44.1 \mathrm{kHz}, 48 \mathrm{kHz})$. If there exists a master system with a master reference clock all signals coming from different systems have to be synchronized by sampling frequency conversion systems to the reference system.

Synchronization for nominally the same sampling rates can be achieved by variable delay techniques in combination with fractional sample-delay algorithms. The more common situation of different plesiochronous sample rates needs sample frequency conversions. The all digital approach of a sampling frequency conversion approximates the digital-toanalog conversion of the source signal and then the analog-to-digital conversion with the new sampling rate [4]. First the sampling rate is increased by a factor of $L=2^{w-1}$, where $w$ denotes the number of bits for the representation of the audio sample, and then a resampling with the new output sampling rate is performed.

Such systems make use of multirate signal processing techniques to reduce the hardware and as well the software complexity. The algorithm for a sampling frequency conversion can be performed by a digital signal processor system as shown in Fig. 5. A sampling frequency conversion between $44.1 \mathrm{kHz}$ and $48 \mathrm{kHz}$ in both directions for a two-channel AES/EBU signal can be achieved with two digital signal processors and special interface circuits. The performance and the accuracy of such a system is strongly influenced by jitter which might exist on the irput and output sampling clock. A more severe problem arises in the case of an asynchronous MADI source, where each channel has to be synchronized by a sampling frequency converter (see Fig. 1). The hardware complexity of a MADI sampling frequency converter can be considered as times 56 of a one channel conversion system (Fig. 6). Therefore the sampling rate of the source must be recovered from the MADI data. Although MADI does not carry information about the sampling rate, it is possible to receive MADI data from a source not being connected to the reference clock of the receiving system. The first bit in a MADI word, the Channel Zero Sync bit, is high for the first channel of a sample period. This periodical pulse at the beginning of the sample period can be used to recover the source sampling rate. This input sampling clock 
is used for the input bus shown in Fig. 6 and for the sampling frequency converters. The reference sampling clock is connected to the sampling frequency converters, the output bus and the MADI transmitter.

\section{Conclusions}

FDDI-based MADI transmission links and the hardware complexity of digital audio interfaces have been discussed in the context of all digital distribution systems and audio processing systems. A common master reference clock should be used for synchronization of different digital audio systems avoiding sampling frequency conversion of MADI sources. The outlined interface techniques show the flexibility and effiency of digital audio distribution systems which use MADI transmission links especially in broadcasting and studio environments.

\section{References}

[1] Audio Engineering Society, "AES Recommended Practice for Digital Audio Engineering - Serial Transmission Format for Linearly Represented Digital Audio Data," J. Audio Eng. Soc., Vol. 33, pp. 975-984, 1985

[2] Audio Engineering Society, "AES Recommended Practice for Digital Audio Engineering - Serial Multichannel Digital Audio Interface (MADI)," J. Audio Eng. Soc., Vol. 39, pp. 368-377, 1991

[3] Am7968/Am7969 Transparent Asynchronous Xmitter-receiver Interface Data Sheet, Advanced Micro Devices, Sunnyvale, CA, May 1987

[4] R.E. Crochiere, L.R. Rabiner, Multirate Digital Signal Processing, Prentice-Hall, Englewood Cliffs, 1983 


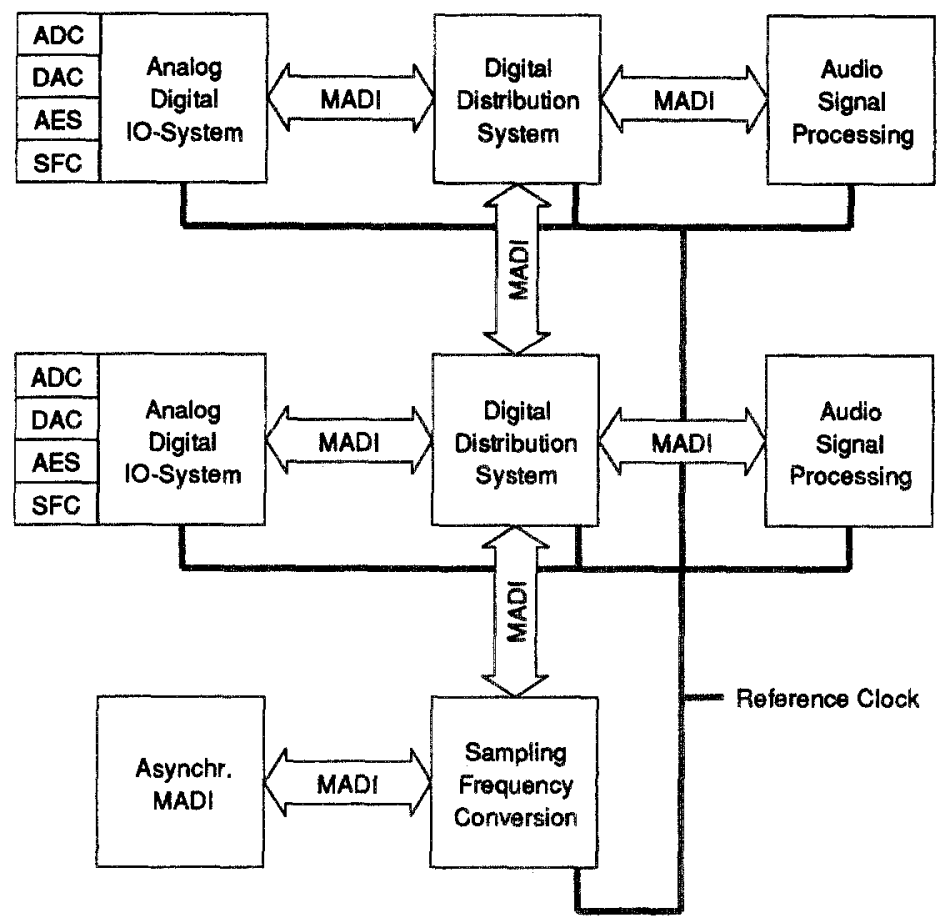

Figure 1: FDDI-based digital audio distribution system

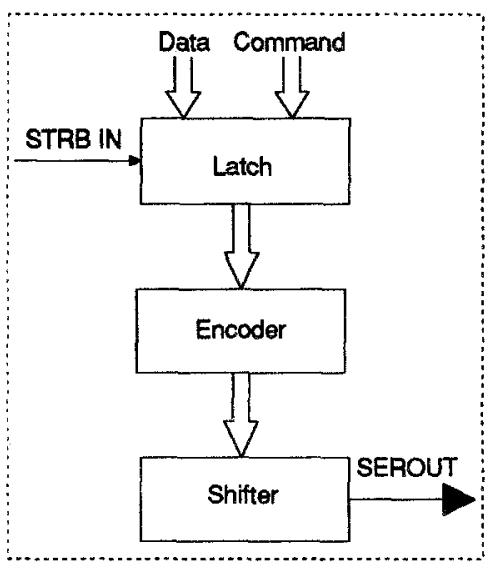

Am7968 (Transmitter)

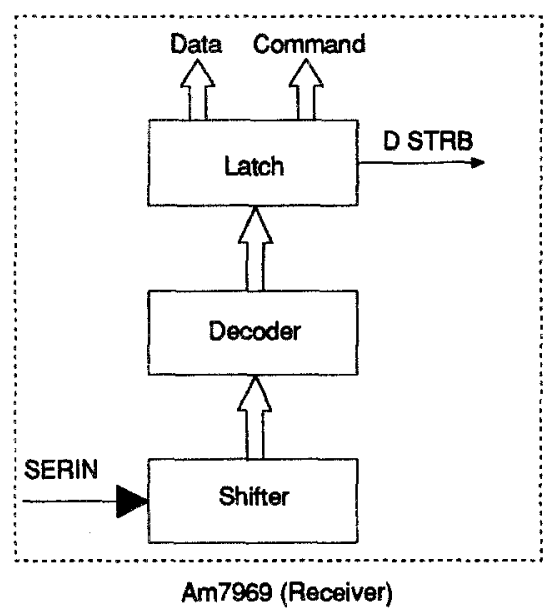

Am7969 (Receiver)

Figure 2: TAXI-Chips 
AES/EBU Format Subtrame :

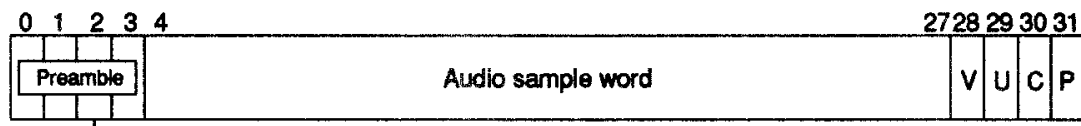

L MADI SYNC

MADI AB

MADI ACTIVE

MADI CHANNEL 0

MADI Frame Period :

\begin{tabular}{|||c|c|c|c|c|}
\hline & channel 0 & channel 1 & channel 54 & channel 55 \\
\hline
\end{tabular}

Figure 3: MADI channel-/sampleframe

\section{TDM-Bus}

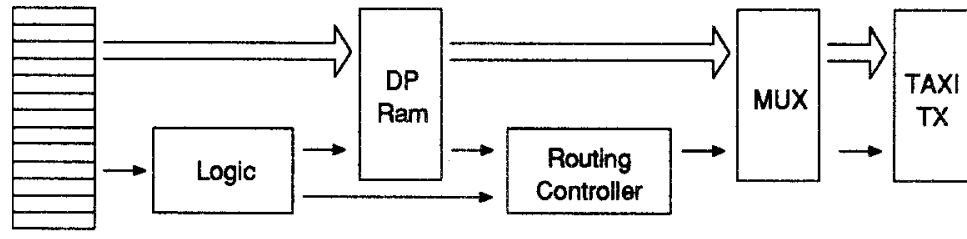

TDM-Bus

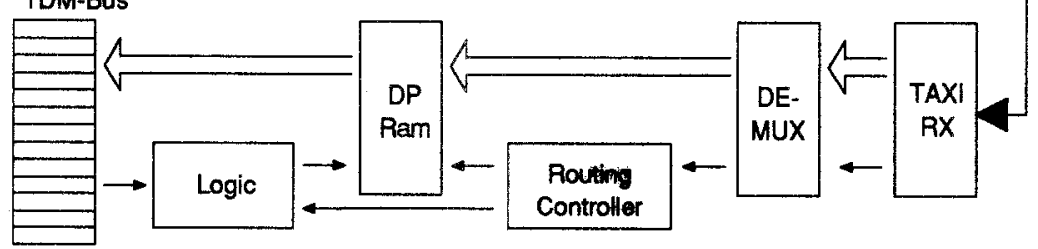

Figure 4: TDM $\rightarrow$ MADI 


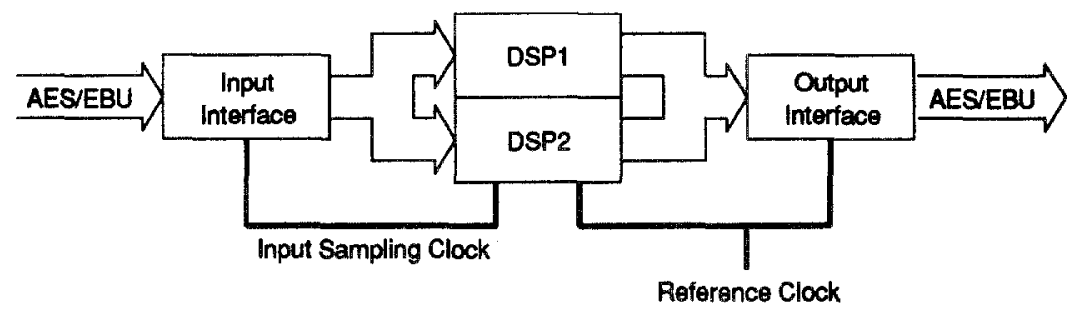

Figure 5: Hardware for two-channel sampling frequency conversion

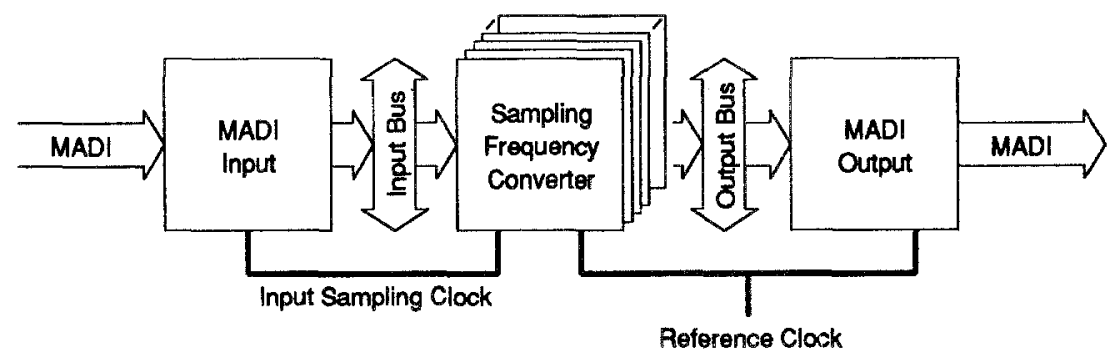

Figure 6: Hardware for MADI sampling frequency conversion 